\title{
Characteristic analysis of a photon converter in a medical LINAC using GEANT4
}

\author{
Junho Ko${ }^{1}$, Beom Yeong Kim², Yoon Sang Kim ${ }^{3}$ \\ ${ }^{1,2,3}$ School of Computer Science and Engineering, Korea University of Technology and Education, \\ Cheonan, Republic of Korea \\ ${ }^{3}$ Institute for Bioengineering Application Technology, Korea University of Technology and Education, \\ Cheonan, Republic of Korea \\ ${ }^{3}$ Corresponding author \\ E-mail:17ich126@koreatech.ac.kr, ${ }^{2}$ tibyte@koreatech.ac.kr,3yoonsang@koreatech.ac.kr
}

Received 12 September 2017; accepted 19 September 2017

DOI https://doi.org/10.21595/vp.2017.19169

Check for updates

Abstract. Medical LINACs (Linear Accelerators) use high energy photon beams to treat deeply located tumors. Generally, neutrons are produced by photonuclear reactions in the head of a medical LINAC using photon beams over $8 \mathrm{MV}$. Neutrons with high relative biological effectiveness and high permeability can cause many problems in terms of radiation protection. The threshold energy of photons producing neutrons in the head of a medical LINAC is $13.06 \mathrm{MV}$ (Aluminum), 13.38 MV (Iron), 8.06 MV (Gold), and 7.41 MV (Tungsten). In this paper, we aim to analyze the characteristics of a photon converter in a medical LINAC using GEANT4 (Geometry and Tracking). For this, the photon and neutron spectrums of the optimized photon converter with tungsten were simulated with 2-24 MeV electron beams, and the ratio of photons with energies over 7.41 MV was analyzed.

Keywords: photon converter, photon target, medical LINAC, GEANT4.

\section{Introduction}

Medical LINAC (linear accelerator), which is an equipment used in radiotherapy, utilizes a high energy photon beam to treat deeply located tumors. Generally, neutrons are produced by photonuclear reactions in the head of the medical LINAC using photon beams over $8 \mathrm{MV}$ [1]. Neutrons with high relative biological effectiveness (RBE) and permeability can cause many problems in terms of radiation protection [2]. The threshold energies of photons producing neutrons in the head of a medical LINAC are 13.06 MV (Aluminum), 13.38 MV (Iron), 8.06 MV (Gold), and 7.41 MV (Tungsten) [3].

The medical LINAC used in clinics cannot fundamentally block the production of photoneutrons because of the electron acceleration principle and their physical characteristics. Therefore, a study is required to analyze the characteristics and effects of photoneutrons. However, it is difficult to measure neutrons in clinics because their energy is distributed continuously and it is hard to separate them from other secondary particles [4]. The GEANT4 simulation can calculate the particle transport by considering the detailed characteristics of the material and can predict the dose distribution with higher accuracy than the conventional dose calculation algorithm. Furthermore, the scope of its application to the medical physics field is expanding [5] and the reliability of the simulation has been verified [6].

In this paper, we aim to analyze the characteristics of a photon converter in a medical LINAC using GEANT4 (Geometry and Tracking). For this, the photon and neutron spectrums of the optimized photon converter [7] with tungsten were simulated in 2 24 MeV electron beams, and the ratio of the photons with energies over 7.41 MV was analyzed. Furthermore, we analyzed the ratio of photons with energies over 7.41 MV that can produce neutrons.

\section{Design of GEANT4 simulation environment converter and photon converter}

An electron incident on a target loses its kinetic energy, which is moved until it comes to a stop in the material. Photon transport induces a bremsstrahlung spectrum with the maximum 
energy of the electron beam. The factors influencing the bremsstrahlung cross-sectional area are the energy of the electron beam, the atomic number of the target material, and the thickness of the target [8]. The optimized photon converter [7] is made of tungsten, and is cylindrical in shape with a radius of $1.5 \mathrm{~cm}$ and thickness of $1 \mathrm{~mm}$ as shown in Fig. 1.

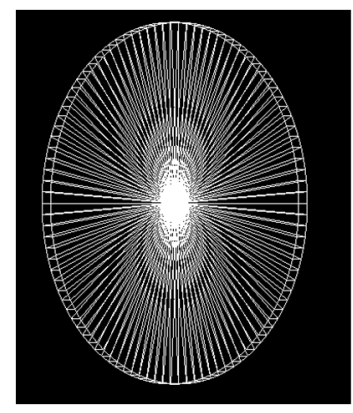

a) The designed photon converter

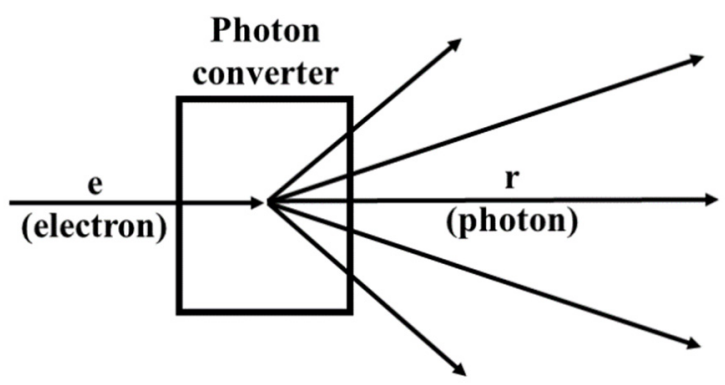

b) The concept of the photon converter

Fig. 1. The concept and the design of the photon converter

GEANT4 9.10 p02 [9] was used for the simulation to analyze the characteristics of the optimized photon converter. The cross-sectional data and the particle transport method were sourced from GEANT4 physics reference manual [9], and materials are defined by NIST [10]. The bremsstrahlung model utilizes the Begert and Seltzer model [11]. During the simulation, the electron beam was vertically incident on the photon converter at a position $2 \mathrm{~mm}$ away from the center point of the incidence plane. The simulation used 107 electron beams to minimize the statistical uncertainty. Fig. 2 shows a GEANT4 simulation result screenshot for electron beams incident on the photon converter.

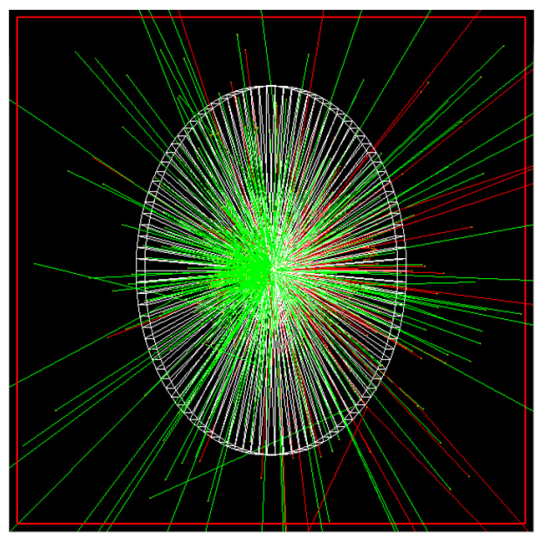

Fig. 2. GEANT4 simulation result screenshot

\section{Analysis of GEANT4 simulation results and photon converter characteristics}

Fig. 3 shows the photon yield spectra of the photon converters for 2, 4, 6, 8, 10, 12, 14, 16, 18, 20,22 , and $24 \mathrm{MeV}$ electron beams. The characteristic radiation at all beam energies was produced at the $520 \mathrm{kV}$. The results are due to the characteristics of the photon, which is less affected by the characteristic radiation in photon beam over $300 \mathrm{kV}$. Furthermore, it was observed that the characteristic radiation appears at relatively high energy only $520 \mathrm{kV}$ because the characteristic radiation with low energy is absorbed in the photon converter.

Table 1 shows the results of the analysis of the photon beam energy spectrum, which serves as a reference for investigating the photoneutrons produced by photonuclear reactions during the interactions between photons and matter. The number and content ratios of photons obtained over 
7.41 MV are provided against the cumulative number of measured photons at $1 \mathrm{~cm}$ below the photon converter.

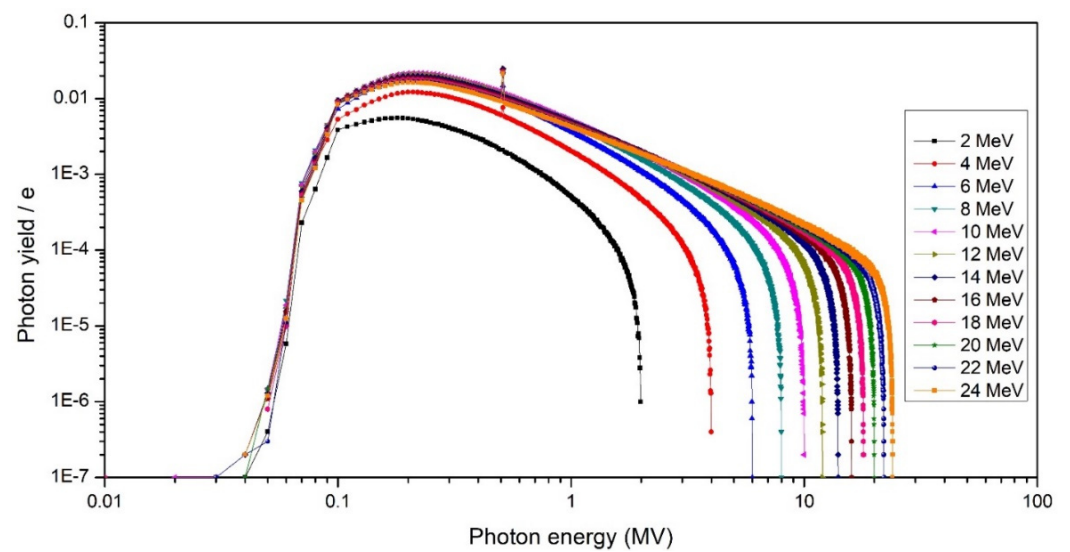

Fig. 3. Photon yield spectrum of photon converter for different electron beam energies

Table 1. Photon yield of photon converter in different electron beam energy

\begin{tabular}{|c|c|c|}
\hline \multirow{2}{*}{ Electron beam energy $(\mathrm{MeV})$} & \multicolumn{2}{|c|}{ Photon yield / e } \\
\cline { 2 - 3 } & For all energies & over 7.41 MV \\
\hline 2 & $0.4512(100 \%)$ & $0(0 \%)$ \\
\hline 4 & $1.2187(100 \%)$ & $0(0 \%)$ \\
\hline 6 & $1.9564(100 \%)$ & $0(0 \%)$ \\
\hline 8 & $2.3705(100 \%)$ & $0.0006(0.02 \%)$ \\
\hline 10 & $2.4996(100 \%)$ & $0.0130(0.52 \%)$ \\
\hline 12 & $2.4738(100 \%)$ & $0.0385(1.55 \%)$ \\
\hline 14 & $2.3948(100 \%)$ & $0.0685(2.86 \%)$ \\
\hline 16 & $2.3130(100 \%)$ & $0.0981(4.24 \%)$ \\
\hline 18 & $2.2526(100 \%)$ & $0.1255(5.57 \%)$ \\
\hline 20 & $2.2143(100 \%)$ & $0.1515(6.84 \%)$ \\
\hline 22 & $2.1903(100 \%)$ & $0.1756(8.01 \%)$ \\
\hline 24 & $2.1815(100 \%)$ & $0.1983(9.09 \%)$ \\
\hline
\end{tabular}

Fig. 4 shows the neutron yield spectra of the photon converters at 2, 4, 6, 8, 10, 12, 14, 16, 18, 2022 , and $24 \mathrm{MeV}$ electron beams. The maximum neutron yield was measured as 5.0-7 and 6.7-7 neutron yield/e at $10 \mathrm{MeV}$ and $22 \mathrm{MeV}$ electron beams respectively.

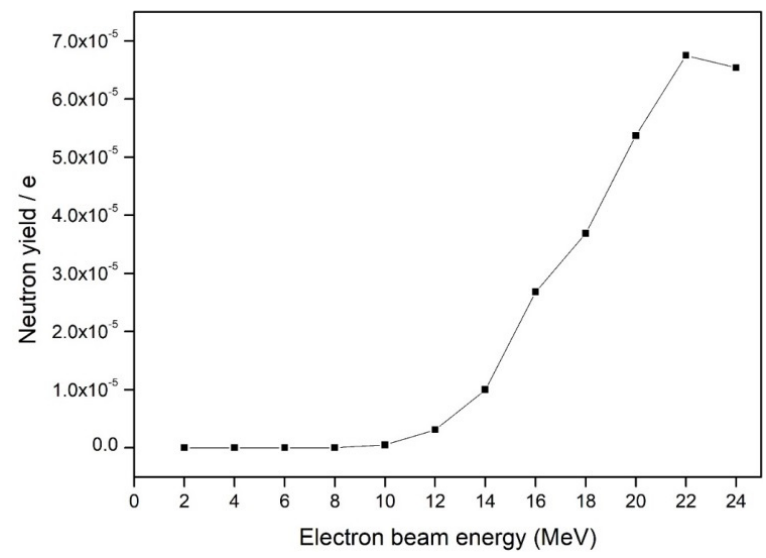

Fig. 4. The neutron yield of the photon converter for different electron beam energies 


\section{Conclusions}

The medical LINAC cannot block the production of photoneutrons because of the electron acceleration principle and their physical characteristics. Therefore, this paper proposes basic data regarding radiation protection by analyzing the characteristics of photoneutrons. To perform this analysis, we modeled the photon converter in the medical LINAC head using GEANT4, which is a Monte Carlo simulation. Simulations were performed to analyze the photoneutron characteristics at 2-24 MeV electron beams, which are typically used in clinics.

In the simulation results, we confirmed that the characteristic radiation is observed at $520 \mathrm{kV}$ and the ratio of photons obtained with energy over 7.41 MV increased as the electron energy increased. Furthermore, the neutron. generation from the photon was maximum at $10 \mathrm{MeV}$ electron beam, and the production of neutrons increased as the electron energy increased. As the neutrons have a high RBE, even low doses can be harmful to the patient. There is also the problem of causing exposure to the operator by radiating to the surrounding environment. Therefore, the neutrons generated from the photon converters must be considered when designing the shields. The results of this study are expected to be used to improve medical LINACs by solving these problems.

\section{Acknowledgement}

This research was supported by Basic Science Research Program through the National Research Foundation of Korea (NRF) funded by the Ministry of Education (No. 2016R1D1A1B03934505).

\section{References}

[1] Khan F. M. The Physics of Radiation Therapy. Lippincott Williams and Wilkins, 2010.

[2] Chibani O., Ma C. M. C. Photonuclear dose calculations for high-energy photon beams from Siemens and Varian linacs. Medical Physics, Vol. 30, 2003, p. 1990-2000.

[3] Olsen K. J. Neutron Contamination from Medical Electron Accelerators (NCRP Report No. 79). Medical Physics, Vol. 1, Issue 3, 1986, p. 968-969.

[4] Becker J. Simulation of Neutron Production at a Medical Linear Accelerator. Doctoral Dissertation, Department of Physics, University of Hamburg, performed at the University Medical Center HamburgEppendorf, Department of Radiotherapy and Radio-Oncology, Medical Physics, 2007.

[5] Kang S. K., Kim C. Y. Structure and application of GEANT4 in radiation science. Journal of Radiation Industry, Vol. 1, 2007, p. 39-46.

[6] ISO/IEC JTC1, Joint Technical Committee 1. ISO/IEC DTR 15504 Software Process Assessment, 2007.

[7] Kim Y. S., Khazaei Z., Ko J., Afarideh H., Ghergherehchi M. Estimation of photoneutron yield in linear accelerator with different collimation systems by Geant 4 and MCNPX simulation codes. Physics in Medicine and Biology, Vol. 61, 2016, p. 2762-2779.

[8] Koch H. W., Motz J. W. Bremsstrahlung cross-section formulas and related data. Reviews of Modern Physics, Vol. 31, 1959, p. 920-955.

[9] GEANT4 Physics Reference Manual. Available: http://GEANT4.web.cern.ch.

[10] Amako K., Guatelli S., Ivanchencko V., Maire M., Mascialino B., Murakami K., Sasaki T. Geant4 and its validation. Nuclear Physics B - Proceedings Supplements, Vol. 150, 2006, p. 44-49.

[11] Arce P., Lagares J. I., Harkness L., Pérez-Astudillo D., Cañadas M., Rato P., Diaz A. Gamos: A framework to do Geant 4 simulations in different physics fields with a user-friendly interface. Nuclear Instruments and Methods in Physics Research Section A, Vol. 735, 2014, p. 304-313. 